\title{
The Tissue Issue: When to Biopsy Persistent or Refractory Disease in Classic Hodgkin Lymphoma
}

\author{
Devi Sampat Gaurav Goyal \\ Division of Hematology-Oncology, University of Alabama at Birmingham, Birmingham, AL, USA
}

Classic Hodgkin lymphoma (cHL) is well known to be a highly curable disease, with 5-year survival rates above $80 \%$. Therefore, accurate diagnosis and staging are imperative, as they may have a significant impact on management. Although the treatment protocols for $\mathrm{cHL}$ can vary across pediatric and adult populations, the disease biologies for both age groups are similar, as is the diagnostic workup. cHL is largely confined to the lymph nodes at diagnosis, and the spread of disease to bones is rare, with an estimated true cortical bone involvement of up to $8 \%$ in adults and even lower rate in adolescents [1].

The case report by Lapietra et al. [2] is of a 15-year-old female diagnosed with stage $4 \mathrm{cHL}$ who underwent initial standard chemotherapy and further salvage therapy followed by autologous stem cell transplantation (ASCT) due to persistent bone lesions seen on a posttreatment positron emission tomography (PET) computed tomography (CT) scan. Due to persistent uptake in the bones, an eventual bone biopsy was undertaken which revealed fibrous dysplasia instead of malignancy. In retrospect, the patient was likely in complete response after first-line treatment and underwent ASCT for a benign bone disorder with PET avidity. This case highlights the importance of discerning true progression of the disease at restaging

karger@karger.com

(c) 2020 S. Karger AG, Basel

www.karger.com/aha

Karger!
PET scans prior to changing the treatment in any malignancy, especially in a highly curable one like cHL.

Over the last decade, the management of cHL has become increasingly reliant on fluorodeoxyglucose (FDG)PET imaging both in the interim and at the end of treatment. However, response assessment for cHL based on FDG-PET imaging may sometimes lead to false-positive findings and it may need clinical correlation. This is especially true in the setting of mixed responses on scans, with areas of incongruent improvement and progression. Furthermore, if there is a persistent area of disease on scans despite an adequate response at other disease sites (as in this case report), it may warrant a biopsy of the site before intensification of the treatment protocols. Indeed, National Comprehensive Cancer Network guidelines (available at: https://nccn.org/) also support this notion and recommend performing a biopsy on a questionable lesion if posttreatment PET scans show a discordant response.

Multiple studies have reported variable false-positive rates of disease on interim scans. In a recent meta-analysis of 11 lymphoma studies (including 3 pediatric studies) with 139 patients who underwent biopsy of an FDG-avid lesion either during or after the completion of chemo- 
therapy, the false-positive rates were in a broad range (7.7-90.5\%) [3]. When focusing on the subgroup of patients with cHL, the false-positive rate for posttreatment FDG PET-CT was 23.1\%. The reason for false-positive FDG PET imaging may be multifold. One of the reasons false-positive activity may occur on FDG PET scans is that inflammatory and autoimmune conditions are often mistaken for malignancy. London et al. [4] demonstrated the prevalence of sarcoidosis occurring after lymphoma. The study uncovered that sarcoidosis was identified in $53 \%$ of patients with questionable relapse of lymphoproliferative diseases, including 4 of 15 patients with cHL.

Another challenge with the use of FDG-PET based imaging for staging of lymphomas may arise from variable patterns of bone involvement. The radiographic features of osseous lymphoma involvement may be predominantly lytic, sclerotic, or mixed [5]. Furthermore, cortical bone involvement cannot be easily differentiated from marrow involvement on standard FDG PET-CT scans. In order to standardize the radiology criteria for cortical bone involvement in cHL, the Staging Evaluation and Response Criteria Harmonization International Working Group generated a consensus document [6]. In cHL, cortical bone involvement was defined as having a positive FDGPET lesion in addition to either a CT or a technitium-99m scan correlate to distinguish cortical lesions from bone marrow involvement. The group also further specified that nodal masses that extend into bone be considered extra-nodal extension and not to be confused with metastatic stage IV disease.

Why is this all particularly important in the realm of cHL? Given that cHL is such a curable disease, it is crucial to obtain an accurate treatment response assessment be- cause the intervention for refractory disease is high-dose chemotherapy followed by ASCT, which carries a significant risk of morbidity and mortality. Given the rarity and complexity of some unique situations including a discordant response or questionable bone involvement, it is prudent to get input from expert nuclear medicine physicians and hematologists. In a recent study from the USA, the overall mortality for $\mathrm{cHL}$ was higher among centers with a low annual volume of patients compared to those with high volume [7]. Given that cHL is an uncommon malignancy, there may be a role for collaboration with centers with expertise and high volumes of disease. Further studies are needed to ascertain more precise measures of tumor response beyond FDG-PET imaging, such as minimal residual disease detection, in order to improve patient outcomes.

\section{Conflict of Interest Statement}

The authors have no conflict of interests to declare.

\section{Funding Sources}

There are no funding sources for this editorial piece.

\section{Author Contributions}

Gaurav Goyal made substantial contributions to the conception and design of this work, as well as acquisition of data and revision and final approval of this paper, and is accountable for this work. Devi Sampat made substantial contributions to the design of this work, contributed to drafting and revision of the content for final approval, and is accountable for this body of work.

\section{References}

1 Gaudio F, Pedote P, Niccoli Asabella A, Ingravallo $\mathrm{G}$, Sindaco $\mathrm{P}$, Alberotanza V, et al. Bone Involvement in Hodgkin's Lymphoma: Clinical Features and Outcome. Acta Haematol. 2018;140(3):178-82.

2 Lapietra G, Moleti L, Giona F, Turchetti A, Celli M, Corsi A et al. Polyostotic fibrous dysplasia mimicking bone Iivolvement in Hodgkin lymphoma: a pediatric case and literature review. Acta Haematol. 10.1159/000508261.

3 Hugo A, Thomas C. Proportion of false-positive lesions at interim and end-of-treatment PDG-PET in lymphoma as determined by histology: systematic review and meta-analy- sis. Eur J Radiol. 85 (2016) 1963-1970. British Institute of Radiology. doi: 10.1259/bjr. 20150980

4 London J, Grados A, Fermé C, Charmillon A, Maurier F, Deau B, et al. Sarcoidosis occurring after lymphoma: report of 14 patients and review of the literature. Medicine (Baltimore). 2014 Nov;93(21):e121.

5 Keraliya AR, Krajewski KM, Jagannathan $J P$, Shinagare AB, Braschi-Amirfarzan $M$, Tirumani SH, et al. Multimodality imaging of osseous involvement In haematological malignancies. Br J Radiol. 2016;89(1059): 20150980 .
6 Lewis J, McCarten K, Kurch L, Flerlage J, Kaste S, Stoevesandt D, et al. Definition of cortical bone involvement in the staging of newly diagnosed pediatric Hodgkin lymphoma: a report from the International Working Group on Staging Evaluation and Response Criteria Harmonization (SEARCH). Pediatr Blood Cancer. doi: 10.1002c/pbc.28142.

7 Goyal G, Tella H, Funni S, Kommalapati A, Inselman J, Shah $\mathrm{N}$, et al. Association between facility volume and mortality of patients with classic Hodgkin lymoma. Available from: https://doi.org/https://doi.org/10.1002/ cncr.32584. 